\title{
A Parallel Derivation to the Maxwell-Garnett Formula for the Magnetic Permeability of Mixed Materials
}

\author{
Hsien-Ming Chang ${ }^{1,2 *}$, Chungpin Liao ${ }^{1,2,3}$ \\ ${ }^{1}$ Advanced Research \& Business Laboratory (ARBL), Taichung, Taiwan (China); ${ }^{2}$ Chakra Energetics, Inc., Incubator, National For- \\ mosa University (NFU), Huwei, Taiwan (China); ${ }^{3}$ Graduate School of Electro-Optic and Material Science, National Formosa Uni- \\ versity (NFU), Huwei, Taiwan (China). \\ Email: hmchuwei@yahoo.com.tw, cpliao@alum.mit.edu
}

Received January $4^{\text {th }}, 2011$; revised April $7^{\text {th }}, 2011$; accepted April 10 ${ }^{\text {th }}, 2011$.

\begin{abstract}
Although mixing formulas for the effective-medium type of approximations for the dielectric permittivities in the infi-nite-wavelength (i.e., quasistatic) limit, such as the Maxwell Garnett formula, have been popularly applied in the whole spectral range of electromagnetic fields, their magnetic counterpart has seldom been addressed up to this day. An effort is thus devoted to the derivation of such an equation to predict the final permeability as the result of mixing together several materials. In a similar fashion to the approach leading to the Maxwell Garnett formula, a model is adopted wherein an originally isotropic host material is embedded with a cluster of spherical homogeneous magnetic particles. It is expected that such obtained formula should find wide applications, and particularly in the light frequency domain in this blossomful era of nanometer technology.
\end{abstract}

Keywords: Mixing Formula, Magnetic Permeability, Maxwell Garnett Mixing Formula

\section{Introduction}

Macroscopic mixing theories have been effectively applied in predicting the approximate would-be dielectric properties of the final mixtures without having to deal with the microscopic fields in an in-situ manner. In the infinite-wavelength approximation, or the socalled "quasistatic limit," the effective electromagnetic characteristics of mixtures can be properly described by two independent quantities, i.e., a single effective permittivity and a single effective permeability, to their own entirety. Although it was shown that in the finitewavelength limit (or, termed the "long-wave-length limit" by many, e.g., [1]) such kind of partition is inapplicable, the interest of this current work is in situations where the mixing of materials is in a practically homogeneous manner and thus the infinite-wavelength approximation should suffice.

However, although mixing formulas for the effective-medium type of approximations for the dielectric permittivities in such infinite-wavelength (i.e., quasistatic) limit, such as the Maxwell Garnett formula [2], have been popularly applied in the whole spectral range of electromagnetic fields, their magnetic counterpart has seldom been addressed up to this day. The current effort is thus to derive such an equation to approximately predict the final permeability as the result of mixing together several magnetic materials.

It should be noticed that, unlike Sheng's et al. [3] approach where there are one or several major permanent magnetic moments, this work is aiming at situations where no such major permanent magnetization exists. In addition, following Maxwell Garnett's [2] model in which a host material contained a collection of spherical homogeneous inclusions, Bruggeman [4] further extended the mixing equation to a more convenient form in which the included particles no long serve as mere perturbation to the host. Since then, various mixing formulas have found a great variety of applications in the prediction of mixed dielectric properties $[5,6]$.

Nevertheless, the situation is quite different when it comes to predicting mixed magnetic permeabilities. Namely, not only the means to calculate magnetic permeability are far from rigorous among even the existing $1^{\text {st }}$ - principle quantum mechanical software (see, 
e.g., [7], wherein the relative permeability is essentially set to unity), but also the crude macroscopic clues to follow, like Maxwell Garnett or Bruggeman formulas for dielectric, are hard to come by. Thus, at least macroscopically, the authors intend to bring back the due balance among the conjugate worlds of dielectrics and magnetics by basing their derivation on a similar model adopted by Maxwell Garnett and Bruggeman [2,4].

\section{A Brief Review of the Derivation Leading to the Maxwell Garnett and Bruggeman Formulas}

Historically, an isotropic host material was hypothesized to embed with a collection of spherical homogeneous inclusions. With the molecular polarization of a single molecule of such inclusions being denoted $\alpha$, the following relation was established within the linear range [8]:

$$
\vec{p}_{m}=\alpha \varepsilon_{0} \vec{E}_{m}
$$

where $\vec{p}_{m}$ was the induced dipole moment and $\vec{E}_{m}$ was the polarizing electric field intensity at the location of the molecule. Since the treatment was aiming for uniform spherical inclusions, the polarizability became a scalar, such that $\vec{E}_{m}$ was expressed as [9]:

$$
\vec{E}_{m}=\vec{E}+\vec{E}_{p}+\vec{E}_{\text {near }}
$$

Here $\vec{E}$ was the average field within the bulk host, $\vec{E}_{p}$ was the electric field at this molecular location caused by all surrounding concentric spherical shells of the bulk, and $\vec{E}_{\text {near }}$ was due to asymmetry within the inclusion. In those cases of interest where either the structure of the inclusion was regular enough, such as a cubical or spherical particulate, or all incorporated molecules were randomly distributed, $\vec{E}_{\text {near }}$ became essentially zero. It was further approximated that $\vec{E}_{m}=\vec{E}+\vec{P} /\left(3 \varepsilon_{0}\right)$ [9], to be elaborated later, with $\vec{P}$ being the polarization density associated with a uniformly polarized sphere, and $\varepsilon_{0}$ being the permittivity in free space. Hence, given (1), with the number density of such included molecules denoted as $n$, and $\vec{P}=n \vec{p}_{m} \quad$ [8], the polarization density was further expressed as:

$$
\vec{P}=n \alpha \varepsilon_{0}\left[\vec{E}+\vec{P} /\left(3 \varepsilon_{0}\right)\right]
$$

However, it was well-known that for isotropic media $\vec{P}=\left(\varepsilon_{r}-1\right) \varepsilon_{0} \vec{E}$ where $\varepsilon_{r}$ stood for the relative permittivity (i.e., the electric field at the center of a uniformly polarized sphere (with $\vec{P}$ being its polarization density) was $\left.-\vec{P} /\left(3 \varepsilon_{0}\right)\right)$. Then, a relation known as the LorentzLorenz formula readily followed $[10,11]$ :

$$
\alpha=\frac{3\left(\varepsilon_{r}-1\right)}{n\left(\varepsilon_{r}+2\right)}
$$

In those special cases where the permittivity of each tiny included particle was $\varepsilon_{s}$ and the host material was vacuum $\left(\varepsilon_{r}=1\right)$, such that $n=V^{1}$ ( $V$ being the volume of the spherical inclusions), and (4) would have to satisfy [2]:

$$
\alpha=3 V \frac{\varepsilon_{s}-\varepsilon_{0}}{\varepsilon_{s}+2 \varepsilon_{0}}
$$

Combining (4) and (5) gave the effective permittivity $\left(\varepsilon_{e f f}\right)$ of the final mixture [2]:

$$
\varepsilon_{e f f}=\varepsilon_{r} \varepsilon_{0}=\varepsilon_{0}+3 f \varepsilon_{0} \frac{\varepsilon_{s}-\varepsilon_{0}}{\varepsilon_{s}+2 \varepsilon_{0}-f\left(\varepsilon_{s}-\varepsilon_{0}\right)}
$$

with $f=n V$ being the volume ratio of the embedded tiny particles $(0 \leq f \leq 1)$ within the final mixture. If, instead of vacuum, the host material was with a permittivity of $\varepsilon_{h}$, (6) was then generalized to the famous Maxwell Garnett mixing formula:

$$
\varepsilon_{e f f}=\varepsilon_{h}+3 f \varepsilon_{h} \frac{\varepsilon_{s}-\varepsilon_{h}}{\varepsilon_{s}+2 \varepsilon_{h}-f\left(\varepsilon_{s}-\varepsilon_{h}\right)}
$$

For the view in which the inclusion was no longer treated as a perturbation to the original host material, Bruggeman managed to come up with a more elegant form wherein different ingredients were assumed to be embedded within a host [4]. By utilizing (4) and (5), he had:

$$
\frac{\varepsilon_{\text {eff }}-\varepsilon_{0}}{\varepsilon_{\text {eff }}+2 \varepsilon_{0}}=\sum_{i} f_{i} \frac{\varepsilon_{i}-\varepsilon_{0}}{\varepsilon_{i}+2 \varepsilon_{0}}
$$

where $f_{i}$ and $\varepsilon_{i}$ are the volume ratio and permittivity of the $i$-th ingredient.

\section{The Magnetic Flux Density at the Center of a Uniformly Magnetized Sphere}

In the above, the adopted relation leading to the Maxwell Garnett mixing formula, i.e., $\vec{E}_{m}=\vec{E}+\vec{P} /\left(3 \varepsilon_{0}\right)$, was achieved by noting that the anti-reactive electric field at the center of a uniformly polarized sphere (see, Figure 1) was $-\vec{P} /\left(3 \varepsilon_{0}\right)$. This result can be derived from integrating the positive surface charges on the upper sphere and negative surface charges on the lower sphere (see Figure 1) [9].

In a similar fashion, surface current can be expected to appear on the surface of a uniformly magnetized sphere (wherein $\vec{M}$ is the finalized net anti-responsive magnetization vector, see Figure 2).

In Figure 2, $\vec{K}_{S}$ is the induced anti-reactive surface current density (in $\mathrm{A} / \mathrm{m}$ ) on the sphere's surface and is equal to:

$$
\vec{K}_{S}=\vec{M} \times \hat{r}=\hat{\phi} M \sin \theta
$$

where $\hat{r}, \hat{\theta}$ and $\hat{\phi}$ are the three orthogonal base vectors of the spherical coordinate. According to the BiotSavart law, the differential magnetic flux density at the 


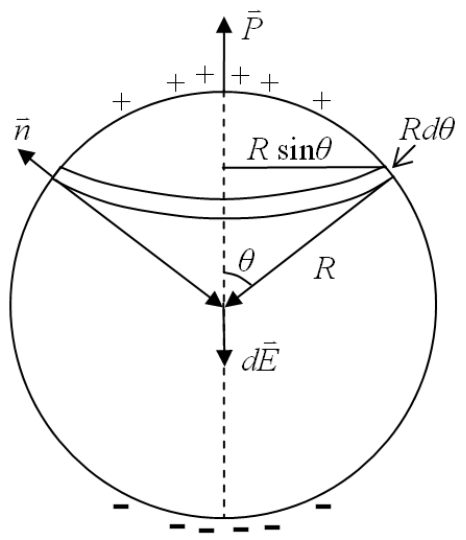

Figure 1. Situation for calculation of the central electric field on a uniformly polarized sphere.

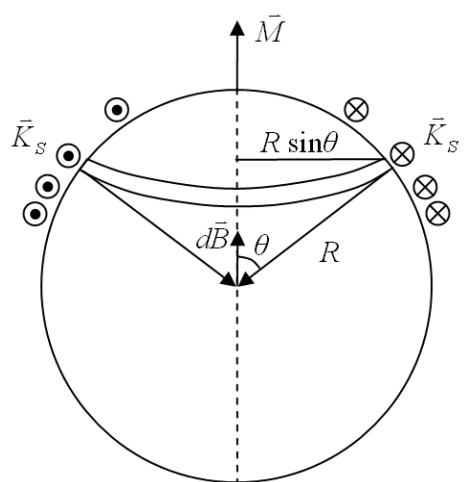

Figure 2. Situation for calculation of the central magnetic flux density on a uniformly magnetized sphere.

sphere's center owing to the surface current on a strip of width $R \mathrm{~d} \theta$ is:

$$
\mathrm{d} \vec{B}=\frac{\mu_{0} I}{4 \pi} \oint_{C} \frac{\mathrm{d} \vec{l} \times(-\hat{r})}{R^{2}}=-\frac{\mu_{0} I}{4 \pi} \oint_{C} \frac{\hat{\theta} \mathrm{d} l}{R^{2}}
$$

where $I \mathrm{~d} \vec{l}$ is the current element on an infinitesimal segment of the strip and $I=K_{S} R \mathrm{~d} \theta . C$ is the path of the strip closing around the north-pointing axis and $\mu_{0}$ the permeability in free space. With all components cancelling one another except the ones parallel to the north-pointing axis, the net induced magnetization density $\vec{M}$ finally results, as do the associated magnetic flux density $\vec{B}$. By integrating all strips on the sphere's surface the magnetic flux density $\left(\vec{B}_{c}\right)$ at the center of a uniformly magnetized sphere is obtained to be [12]:

$$
\vec{B}_{c}=\frac{2 \vec{M} \mu_{0}}{3}
$$

\section{The Mixing Formula for Magnetic Permeabilities}

Now, this time consider an isotropic host material embedded with a collection of spherical homogeneous mag- netic particles. Given the magnetic flux density at the location of a single molecule of the inclusions being $\vec{B}_{m}$, the following relation holds in general:

$$
\vec{B}_{m}=\vec{B}-\vec{B}_{c}+\vec{B}_{\text {near }}
$$

where $\vec{B}$ is the average magnetic flux density within the bulk host and $\vec{B}_{\text {near }}$ is due to the asymmetry in the inclusion. In those cases of interest where either the structure of the included particles is regular enough, such as a cubical or spherical particulate, or all incorporated molecules are randomly distributed, $\vec{B}_{\text {near }}$ can be taken as zero.

If the magnetic field intensity at the location of the molecule is denoted $\vec{H}_{m}$, the induced magnetic dipole moment $\left(m_{m}\right)$ is:

$$
\vec{m}_{m}=\kappa_{m} \vec{H}_{m}
$$

where $\kappa_{m}$ is the molecular magnetization of the molecule. Because $\vec{M}$ equals $n \vec{m}_{m}$, we have [8]

$$
\vec{M}=n \kappa_{m} \vec{H}_{m}=\chi_{m} \vec{H}_{m}
$$

where $\chi_{m}$ is known as the magnetic susceptibility. Hence, $\bar{B}_{m}$ can be further expressed as [8]

$$
\vec{B}_{m}=\mu_{0} \mu_{r} \vec{H}_{m}=\mu_{0}\left(1+n \kappa_{m}\right) \vec{H}_{m}=\mu_{0}\left(1+\chi_{m}\right) \vec{H}_{m}
$$

with $\mu_{r}$ being the relative permeability. By incorporating (12) and (15) into (14) we obtain:

$$
M=\frac{n \kappa_{m}}{\mu_{0}\left(1+n \kappa_{m}\right)}\left(B-\frac{2 M \mu_{0}}{3}\right)
$$

Further, for isotropic magnetized materials [8]:

$$
\begin{gathered}
M=\left(\mu_{r}-1\right) H=\frac{\left(\mu_{r}-1\right) B}{\mu_{0} \mu_{r}}, \text { or } \\
B=\frac{\mu_{0} \mu_{r}}{\left(\mu_{r}-1\right)} M
\end{gathered}
$$

Substituting (17) into (16) gives

$$
\kappa_{m}=\frac{3}{n}\left(\frac{\mu_{r}-1}{-2 \mu_{r}+5}\right)
$$

In the special case where the host material is vacuum $\left(\mu_{r}=1\right)$ and the permeability of the spherical particles is $\mu_{s}, n=V^{1}$ ( $V$ being the volume of a spherical particle), and (18) is satisfied by:

$$
\kappa_{m}=3 V \frac{\mu_{s}-\mu_{0}}{-2 \mu_{s}+5 \mu_{0}}
$$

Combining (18) and (19) gives the effective permeability $\left(\mu_{e f f}\right)$ of the final mixture, i.e.,

$$
\mu_{e f f}=\mu_{r} \mu_{0}=\mu_{0}+3 f \mu_{0} \frac{\mu-\mu_{0}}{-2 \mu+5 \mu_{0}+2 f\left(\mu-\mu_{0}\right)}
$$


where $f=n V$ is the volume ratio of the embedded particles within the mixture $(0 \leq f \leq 1)$. In the more general situations where the host is no longer vacuum but of the permeability $\mu_{h}$, then the more general mixing formula of permeabilities becomes:

$$
\mu_{e f f}=\mu_{h}+3 f \mu_{h} \frac{\mu_{s}-\mu_{h}}{-2 \mu_{s}+5 \mu_{h}+2 f\left(\mu_{s}-\mu_{h}\right)}
$$

As with Bruggeman's approach for dielectrics [4], the derived magnetic permeability formula can be generalized to the multi-component form:

$$
\frac{\mu_{e f f}-\mu_{0}}{-2 \mu_{e f f}+5 \mu_{0}}=\sum_{i} f_{i} \frac{\mu_{i}-\mu_{0}}{-2 \mu_{i}+5 \mu_{0}}
$$

where $f_{i}$ and $\mu_{i}$ denote the volume ratios and permeabilities of the involved different inclusions, respectively. Or,

$$
\frac{\mu_{r e f f}-1}{-2 \mu_{r e f f}+5}=\sum_{i} f_{i} \frac{\mu_{r i}-1}{-2 \mu_{r i}+5}
$$

Although the actual mixing procedures can vary widely such that substantial deviations may result between the theoretical and measured values, (23) should still serve as a valuable guide when designing magnetic materials or composites.

\section{Summary and Conclusions}

An effective mixed medium approximation of permeabilities in the infinite-wavelength limit is derived from the model describing a host material containing a collection of spherical homogeneous inclusions. The derivation parallels that which had previously led to the famous Maxwell Garnett formula for dielectric mixtures. It is believed that such approximate magnetic mixing formula should find a great many applications in all frequency spectra of the general electromagnetic fields.

\section{REFERENCES}

[1] W. Lamb, D. M. Wood and N. W. Ashcroft, "LongWavelength Electromagnetic Propagation in Heterogeneous Media," Physical Review B, Vol. 21, No. 6, March
1980, pp. 2248-2266. doi:10.1103/PhysRevB.21.2248

[2] J. C. Maxwell Garnett, "Colours in Metal Glasses and in Metallic Films," Philosophical Transactions of the Royal Society of London. Series A, Containing Papers of a Mathematical or Physical Character, Vol. 203, 1904, pp. 385-420. doi:10.1098/rsta.1904.0024

[3] Ping Sheng and Mireille Gadenne, "Effective Magnetic Permeability of Granular Ferromagnetic Metals," Journal of Physics: Condensed Matter, Vol. 4, No. 48, 1992, pp. 9735-9740. doi:10.1088/0953-8984/4/48/025

[4] D. A. G. Bruggeman, "Berechnung verschiedener physikalischer Konstanten von heterogenen Substanzen. I. Dielektrizitätskonstanten und Leitfähigkeiten der Mischkörper aus isotropen Substanzen," Annalen der Physik, Vol. 416, No. 7, 1935, pp. 636-664. doi:10.1002/andp.19354160802

[5] J. Jamnik, J. R. Kalnin, E. A. Kotomin and J. Maier, "Generalised Maxwell-Garnett Equation: Application to Electrical and Chemical Transport," Physical Chemistry Chemical Physics, Vol. 8, No. 11, 2006, pp. 1310-1314. doi:10.1039/b514448p

[6] J. I. Gittleman, B. Abeles, "Comparison of the Effective Medium and the Maxwell-Garnett Predictions for the Dielectric Constants of Granular Metals," Physical Review B, Vol. 15, No. 6, March 1977, pp. 3273-3275. doi:10.1103/PhysRevB.15.3273

[7] Cambridge Sequential Total Energy Package (CASTEP) and DMol3, Administered by Accelrys Inc.

[8] D. K. Cheng, "Field and Wave Electromagnetics," Second Edition, Addison-Wesley Pub. Co., Reading, 1989.

[9] E. M. Purcell, "Electricity and Magnetism," Second Edition, Education Development Center, Inc., Massachusetts, 1985.

[10] H. A. Lorentz, "Ueber die Beziehung zwischen der Fortpflanzungsgeschwindigkeit des Lichtes und der Körperdichte," Annalen der Physik, Vol. 245, No. 4, 1880, pp. 641-665.

[11] L. Lorenz, "Ueber die Refractionsconstante," Annalen der Physik, Vol. 247, No. 9, 1880, pp. 70-103.

[12] P. Lorrain and D. R. Corson, "Electromagnetic Fields and Waves," Second Edition, W. H. Freeman \& Co Ltd, San Francisco, 1970. 